\title{
THE POSSIBILITY TO LINK RADIO AND OPTICAL REFERENCE FRAMES WITH ARTIFICIAL SATELLITES
}

\author{
TONG FU \\ Purple Mountain Observatory, \\ Academia Sinica, \\ Nanjing, China.
}

Based on extragalactic radio sources, a new high precision extragalactic radio reference frame can be established from radio interferometric measurements. To link the optical fundamental reference frame presently represented by the FK4/5 to the extragalactic radio frame, the optical counterparts of extragalactic radio sources (quasars, BL Lac objects etc.) and radio stars are the most important classes of objects. Besides these two classes of objects, are there any other objects which can be used to link the optical and radio frames? A posible answer is that artificial satellites could be a candidate class of objects contributing to this subject.

Because of the motion of an artificial satellite on the sky, long-term observation will cover a large area of the sky. From radio observation, the orbit of the satellite can be derived relative to the extragalactic radio frame accurately. The differences between observed optical positions in the optical fundamental frame and radio positions calculated from the theory of orbital motion of the satellite derived from radio observation, $\Delta \alpha(t)$ and $\Delta \delta(t)$, consist of local errors of the optical reference system, the effect of the rotation of the optical frame as well as the errors of the theory of orbital motion of the satellite. If the errors of the theory of orbital motion are small enough, neglecting the local errors of the optical reference system, only the rotation of the optical frame is taken into account, $\Delta \alpha(t)$ and $\Delta \delta(t)$ can be expressed as follows:

$$
\begin{aligned}
& \Delta \alpha(t)=\mathrm{C}_{\alpha}+\mathrm{R}_{\alpha} t \\
& \Delta \delta(t)=\mathrm{C}_{\delta}+\mathrm{R}_{\delta} t
\end{aligned}
$$

where $R_{\alpha}$ and $R_{\delta}$ are the rotation of the optical frame relative to the radio frame, $C_{\alpha}$ and $C_{\delta}$ are the differences of origins of both frames. With long period and large amount of observation, $\mathrm{C}_{\alpha}, \mathrm{C}_{\delta}, \mathrm{R}_{\alpha}$ and $R_{\delta}$ can be determined with high precision, thus the link between both frames can be established.

There are many advantages to link radio and optical frames with satellites. In the case of extragalactic radio sources and radio stars, the structure of the sources and the inconsistency of radio and optical positions are problems to be solved. The faint optical brightness of quasars and the weak radio emission of radio stars make observation difficult. $T$ hese problems do not occur in the case of satellites. They are point objects. Their emission in both the radio and optical domains can be strong enough. The observation is relatively convenient. If the satellite is bright enough-for example, brighter than 10th magnitude-it can be observed with transit type instruments, such as meridian circle and/or astrolable.

On the other hand, for some satellites with large orbital obliquity, such as GPS, $i>60^{\circ}$, the observation of a satellite covers quite a large area of sky. Most of the sky can be covered only with a few satellites, unlike the use of radio sources and radio stars, which need a large number of objects homogeneously distributed on the whole sky. 